\title{
Evaluación de la red de carreteras y su impacto en costos y tiempos de viaje en una región del norte de Colombia
}

\author{
Carlos Arango \\ Grupo de Simulación de tecnologías Industriales, Facultad de Ciencias Básicas, Ingeniería \\ y Arquitectura, Corporación Universitaria del Caribe, Sincelejo - Colombia, \\ carlos.arango@ cecar.edu.co \\ Cesar Vergara \\ Grupo de Simulación de tecnologías Industriales, Facultad de Ciencias Básicas, Ingeniería \\ y Arquitectura, Corporación Universitaria del Caribe, Sincelejo - Colombia, \\ cesar.vergara@ cecar.edu.co
}

\section{RESUMEN}

El transporte es una actividad fundamental dentro de la sociedad, todas las personas a diario se benefician de los servicios de transporte para desarrollar sus actividades cotidianas. Para gestionar los sistemas de transporte en una región geográfica es necesario contar con información técnica de las vías, pero diversos estudios han demostrado que los países Latinoamericanos no cuentan con eficiente información técnica de su red de carreteras. Este problema afecta la sociedad en varios niveles de forma simultanea ya que el transporte moviliza personas pero también los bienes que son consumidos por estas.

El caso Colombiano no es ajeno a la realidad Latinoamericana y en algunas regiones del país el problema es aún mayor ya que la información de muchas vías no corresponde a la información que registra el Ministerio de Transporte. En este trabajo se diagnosticó la red vial del Departamento de Sucre, ubicado en la región norte de Colombia, realizando un análisis del estado del asfalto, señalización, número de carriles y velocidad de conducción. Lo anterior permitió evaluar las carreteras seleccionadas realizando una comparación con la información disponible por el ministerio de transporte, dando como resultado una matriz de inconsistencias entre la información disponible con la recolectada. Finalmente se midió el impacto en los costos y tiempos de viaje que se realizan en esta región del País.

\section{INTRODUCCIÓN}

Dentro de la logística, el transporte es una operación clave para la toma de decisiones, ya que excluyendo los costos por abastecimiento, el transporte tiene la mayor participación en los costos totales, comparado con cualquier otra actividad logística. Se ha visto que el movimiento de carga representa cerca de dos tercios de los costos totales logísticos en las empresas (Ballou, 2004).

Uno de los principales objetivos de la logística empresarial es colocar los productos en el lugar y momento adecuado, para esto se formulan modelos de transporte que considerando la situación actual definen la mejor ruta para transportar las mercancías entre dos puntos, 
esto también es conocido como la planificación del transporte o el plan maestro de transporte. El objetivo de la planificación del transporte es proporcionar pronósticos de la oferta y la demanda de trayectos futuros, permitiendo a los planificadores que desarrollen medidas a corto, mediano y largo plazo asegurando el movimiento constante y eficiente de personas y bienes.

Diversos autores han descrito el proceso de planificación del transporte tales como Miller, J. y Meyer, J. (1984), Gakenheimer, R. (1995) y Dickey, J. (1983) los cuales aunque con algunas diferencias coinciden en las siguientes seis etapas:

1. Análisis de la información y diagnostico

2. Fijación de los objetivos y las metas

3. Diseño de alternativas

4. Evaluación de las alternativas

5. Programación y presupuesto

6. Monitoreo y retroalimentación

La primera etapa (análisis de la información y diagnostico) es una de las etapas más complejas en la planificación del transporte en Colombia debido a la poca información disponible y a los elevados costos en que se incurren al momento de recolectar los datos necesarios para desarrollar los modelos de transporte. Según Ardila, A. (1999) la información disponible en países en desarrollo como Colombia es deficiente o simplemente no existe en algunas zonas. Aunque han pasado 14 años este problema sigue presente en Colombia ya que un estudio realizado por el Banco de Desarrollo de América Latina (CAF) en el 2013, titulado "La infraestructura en el desarrollo integral de América latina" (Barbero, J. 2013) identificó los problemas que más impactan la productividad de los recursos en diferentes áreas de actuación. En el caso del transporte se encontró que entre los principales problemas están los estudios básicos inexistentes o incompletos.

El Departamento de Sucre en el norte de Colombia no es ajeno a esta situación, todo lo contrario, al ser uno de los departamento más pobres del país la situación se agrava debido al descuido del gobierno central y de alguna forma también del departamental. Lo anterior genera efectos negativos en la planificación del transporte tanto para los agentes públicos como privados, la tabla 1 muestra de forma esquemática la relación de causas y efectos de esta problemática. 


\begin{tabular}{|c|c|c|}
\hline $\begin{array}{l}\text { Efecto Indirecto } 1 . \\
\text { Infraestructuras de } \\
\text { transporte sub utilizadas o } \\
\text { desconectadas de la red de } \\
\text { transporte nacional }\end{array}$ & $\begin{array}{l}\text { Efecto Indirecto } 2 . \\
\text { Aumento de los precios en } \\
\text { los fletes para el transporte } \\
\text { de mercancías y pasajeros. }\end{array}$ & $\begin{array}{l}\text { Efecto Indirecto } 3 . \\
\text { Incremento en los tiempos } \\
\text { de viaje por trayecto }\end{array}$ \\
\hline $\begin{array}{l}\text { Efecto Directo1. } \\
\text { Los organismos estatales no } \\
\text { pueden hacer una correcta } \\
\text { planeación de los sistemas } \\
\text { de transporte }\end{array}$ & $\begin{array}{l}\text { Efecto Directo } 2 . \\
\text { Las empresas } \\
\text { transportadoras no pueden } \\
\text { hacer un costeo eficiente de } \\
\text { sus operaciones }\end{array}$ & $\begin{array}{l}\text { Efecto Directo } 3 . \\
\text { Mala programación del } \\
\text { transporte de mercancías y } \\
\text { pasajeros }\end{array}$ \\
\hline \multicolumn{3}{|c|}{$\begin{array}{l}\text { Deficiente información técnica sobre la infraestructura de transporte } \\
\text { intermodal del Departamento de Sucre. }\end{array}$} \\
\hline $\begin{array}{l}\text { Causa Directa } 1 . \\
\text { Deficiente información } \\
\text { técnica de las } \\
\text { infraestructuras logísticas } \\
\text { del Departamento de Sucre } \\
\text { tales como puertos. } \\
\text { Aeropuertos, etc. }\end{array}$ & $\begin{array}{l}\text { Causa Directa } 2 . \\
\text { Deficiente Información } \\
\text { técnica de la Red Vial del } \\
\text { Departamento de Sucre }\end{array}$ & $\begin{array}{l}\text { Causa directa } 3 . \\
\text { Inexistencia de un mapa de } \\
\text { la red de transporte } \\
\text { intermodal del } \\
\text { Departamento de Sucre }\end{array}$ \\
\hline $\begin{array}{l}\text { Causa Indirecta } 1 . \\
\text { Falta de interés del estado } \\
\text { en el desarrollo de estudios } \\
\text { técnicos de las } \\
\text { infraestructuras logísticas } \\
\text { de Sucre. }\end{array}$ & $\begin{array}{l}\text { Causa Indirecta } 2 . \\
\text { Deficiente información para } \\
\text { realizar la planificación del } \\
\text { transporte por parte de las } \\
\text { empresas privadas }\end{array}$ & $\begin{array}{l}\text { Causa Indirecta } 1 . \\
\text { Inexistente información } \\
\text { técnica de las vías e } \\
\text { infraestructuras logísticas } \\
\text { del Departamento de Sucre. }\end{array}$ \\
\hline
\end{tabular}

Tabla 1 - Árbol del problema

En este trabajo se realizó una revisión de la información disponible sobre la infraestructura vial del Departamento de Sucre tanto por entidades nacionales como regionales, se identificó la forma en que se categorizan las vías, variables que se tienen en cuenta y la administración de los diferentes tramos de la red vial. Posteriormente se realizó una validación de la información encontrada, para lo cual se realizó un levantamiento de información primaria que permitió el análisis del estado del asfalto, señalización, número de carriles y velocidad de conducción Lo anterior permitió evaluar las carreteras seleccionadas realizando una comparación con la información disponible por el ministerio de transporte, dando como resultado una matriz de inconsistencias entre la información disponible con la recolectada. Finalmente se concluyó con el impacto en los costos y tiempos de viaje que se realizan en esta región del País.

\subsection{Planificación del transporte intermodal}

El transporte intermodal comúnmente aparece como la mejor opción dentro de la actividad comercial mundial (Reis, V. et al 2012), esto es debido a que no siempre las empresas y los mercados se encuentran en cercanías de los puertos. Entendiendo como transporte intermodal, al empleo de más de un medio para llevar un embarque hasta su destino a través de una red de transporte. Esta red es un conjunto de nodos y arcos, en donde el transporte se origina y termina en los nodos y viaja a través de los arcos (Chopra, 2008). 
Es común encontrar en países en vía de desarrollo como Colombia que la producción y la demanda se encuentre geográficamente cerca, y que la mayor parte de la fuerza de trabajo se encuentre ocupada en la producción agrícola y una baja proporción de esta en las áreas urbanas. Una de las consecuencias de tener un bajo desarrollo de los sistemas de transporte es que el radio de acción de los mercados se limita a la áreas que rodean los puntos de producción, lo cual no estimula la competencia entre estos y por ende afecta de forma negativa aspectos como la innovación asociada a las operaciones de transporte usando herramientas como los Sistemas de información Geográficos (SIG), contrario a países desarrollados donde las herramientas informáticas son utilizadas en la gestión del transporte para la evaluación de rutas y localizaciones (Moreno, G. 2007)

El diseño de una red de transporte afecta el desempeño de este, ya que establece la infraestructura dentro de la cual se toman las decisiones operacionales de transporte. Una red bien diseñada permite diseñar mejores estrategias a un menor costo (Chopra, 2008).

Algunos de los principales elementos de esta infraestructura son los caminos, puertos marítimos, aeropuertos, vías férreas y canales de navegación que se hallan a lo largo de los nodos y arcos de una red de transporte. En la mayoría de los países el gobierno es el responsable o ha tenido un papel significativo en la construcción y administración de estos elementos de infraestructura (Calkins, H. 1990). Es pertinente anotar que dicha infraestructura no tendría sentido por sí sola, sino como elemento que contribuye a la articulación de sistemas más complejos, viendo que en el mundo del transporte actual todo funciona en red, es decir, cada nodo juega un papel determínate en actividad comercial.

Existen diferentes características de las cuales depende la elección de un modo de transporte o una oferta de servicio que incluye un modo de transporte dentro de una red. Según McGinnis (1990) las seis variables claves para elegir un servicio de transporte son:

- Tarifas de flete

- Seguridad o confiabilidad

- Tiempo en tránsito

- Pérdidas, daños, procesamiento de quejas y reclamaciones, y rastreo;

- Consideraciones de mercado del consignatario, y

- Consideraciones del transportista

En algunas situaciones las tarifas de flete son primordiales y pueden llegar a ser concluyentes al momento de la toma de decisión, no obstante, la calidad del servicio, expresada en el tiempo en tránsito (velocidad) y la variabilidad del tiempo en tránsito (confiabilidad), comúnmente siguen siendo el factor determinante al elegir un servicio de transporte (Ballou, 2004). 


\section{LA GESTIÓN DE LA INFRAESTRUCTURA DE TRANSPORTE EN COLOMBIA}

El ministerio de transporte de Colombia según el decreto 2053 de 2003 tiene como objetivo primordial la formulación y adopción de las políticas, planes, programas, proyectos y regulación económica en materia de transporte, transito e infraestructura de los modos de transporte carretero, marítimo, fluvial, Ferrero y aéreo, así como también la regulación técnica en materia de transporte y tránsito de estos modos, excepto el aéreo que está a cargo de la aeronáutica civil (AEROCIVIL) por la ley 105 de 1193 que redistribuye competencias y recursos de la Nación y las entidades territoriales.

Entre las funciones que están a cargo del Ministerio de transporte está la categorización de la red vial nacional de carreteras. Función que se estipula en el Artículo 1 de la ley 1228 y dice "el Ministerio de Transporte será la autoridad que mediante criterios técnicos, determine a que categoría pertenecen las vías". A continuación se especifican las categorías en las cuales se clasifican las vías que se encuentran en Colombia:

- Arterial o de primer orden (Vía Primaria)

- Intermunicipal o de segundo orden (Vía Secundaria)

- Veredal o de tercer orden (Vía Terciaria)

Estas categorías podrán corresponder a carreteras a cargo de la nación, los Departamentos, los distritos especiales y los Municipios. Los términos de cómo se deben de categorizar las vías están fijados mediante la Resolución número 1240 de 2013 del Ministerio de Transporte. En esta se establecen los siguientes criterios técnicos para categorizar las vías que conforman el Sistema Nacional de Carreteras o Red Vial Nacional:

- Funcionalidad de la vía

- Transito promedio diario - TPD

- Diseño y/o características geométricas de la vía

- Población

Cada uno de estos criterios tiene un peso diferente en el momento de categorizar una vía siendo la funcionalidad el más importante con una ponderación de $40 \%$ a diferencia del resto de criterios los cuales representa un $20 \%$. En la tabla 2 se muestra la relación entre las categorías y los criterios técnicos.

Para la gestión y administración de la red vial Colombiana el ministerio de transporte de este País creó dos entidades las cuales son; el Instituto Nacional de Vías INVIAS mediante el decreto 2171 de 1992, que tiene como objeto la administración y gestión de la infraestructura no concesionada de la red vial Nacional de primer y tercer orden así como de la infraestructura férrea, fluvial y marítima. De forma complementaria creo la Agencia Nacional de Infraestructura ANI mediante el decreto 4165 del 2011 que tiene por objeto administrar y gestionar la infraestructura vial concesionada en todos sus modos. Según las 
cifras del Ministerio de Transporte de 2013 la infraestructura vial de Colombia consta de 203.392 Km. Los cuales son gestionados como se muestra en la tabla 3.

\begin{tabular}{|c|c|c|c|c|c|}
\hline \multirow{2}{*}{ Categoría } & \multirow{2}{*}{ Funcionalidad } & \multicolumn{2}{|c|}{$\begin{array}{c}\text { Transito promedio } \\
\text { diario }\end{array}$} & \multirow{2}{*}{$\begin{array}{c}\text { Diseño } \\
\text { geométrico }\end{array}$} & \multirow{2}{*}{ Población } \\
\hline & & $\begin{array}{l}\text { Límite } \\
\text { inferior }\end{array}$ & $\begin{array}{c}\text { Límite } \\
\text { superior }\end{array}$ & & \\
\hline Ponderación & 40 & \multicolumn{2}{|c|}{20} & 20 & 20 \\
\hline \multirow[b]{2}{*}{$\begin{array}{l}\text { Primer Orden } \\
\text { (Primaria) }\end{array}$} & \multirow{2}{*}{$\begin{array}{l}\text { Permite la } \\
\text { comunicación a } \\
\text { nivel Nacional, } \\
\text { conectando } \\
\text { capitales de } \\
\text { Departamentos, } \\
\text { fronteras, puertos } \\
\text { y zonas de } \\
\text { producción. }\end{array}$} & \multirow[b]{2}{*}{$\begin{array}{c}700 \\
\text { VEH/DÍA }\end{array}$} & \multirow[b]{2}{*}{$\begin{array}{c}>=700 \\
\text { VEH/DÍA }\end{array}$} & $\begin{array}{l}\text { Calzada } \\
\text { doble }\end{array}$ & \multirow{2}{*}{$\begin{array}{l}\text { Población de } \\
\text { capitales de } \\
\text { Departament } \\
\text { o, pasos de } \\
\text { frontera y/o } \\
\text { puertos. }\end{array}$} \\
\hline & & & & $\begin{array}{c}\text { Calzada } \\
\text { sencilla }>=\mathrm{a} \\
7.30 \mathrm{~m}\end{array}$ & \\
\hline $\begin{array}{l}\text { Segundo } \\
\text { Orden } \\
\text { (secundaria) }\end{array}$ & $\begin{array}{l}\text { Permite la } \\
\text { comunicación } \\
\text { entre dos o más } \\
\text { municipios o con } \\
\text { una vía de primer } \\
\text { orden. }\end{array}$ & $\begin{array}{c}150 \\
\text { VEH/DÍA }\end{array}$ & $\begin{array}{c}<700 \\
\text { VEH/DÍA }\end{array}$ & $\begin{array}{c}\text { Calzada } \\
\text { sencilla < a } \\
7.30 \mathrm{~m}\end{array}$ & $\begin{array}{l}\text { Cabeceras } \\
\text { Municipales } \\
\text { con más de } \\
15.000 \\
\text { habitantes. }\end{array}$ \\
\hline $\begin{array}{c}\text { Tercer Orden } \\
\text { (Terciaria) }\end{array}$ & $\begin{array}{lr}\text { Permite } & \text { la } \\
\text { comunicación } \\
\text { entre dos o más } \\
\text { veredas de un } \\
\text { municipio o con } \\
\text { una vía de } \\
\text { segundo orden }\end{array}$ & $\begin{array}{c}>=1 \\
\text { VEH/DÍA }\end{array}$ & $\begin{array}{c}<150 \\
\text { VEH/DÍA }\end{array}$ & $\begin{array}{c}\text { Calzada } \\
\text { sencilla }<=\mathrm{a} \\
6 \mathrm{~m}\end{array}$ & $\begin{array}{l}\text { Cabeceras } \\
\text { Municipales } \\
\text { con menos de } \\
15.000 \\
\text { habitantes. }\end{array}$ \\
\hline
\end{tabular}

Tabla 2 - Criterios para categorizar las vías de la red vial Colombiana

\begin{tabular}{|c|c|c|c|c|c|c|c|c|c|c|}
\hline \multirow{3}{*}{ AÑ̃ } & \multicolumn{3}{|c|}{ PRIMARIAS } & \multirow{3}{*}{\begin{tabular}{|c|} 
SECUNDARIAS \\
$\begin{array}{c}\text { A cargo de los } \\
\text { departamentos - } \\
\text { PVR }\end{array}$
\end{tabular}} & \multicolumn{4}{|c|}{ TERCIARIAS } & \multirow{3}{*}{$\begin{array}{l}\text { TOTAL RED } \\
\text { VIAL } \\
\text { NACIONAL }\end{array}$} & \multirow{3}{*}{$\begin{array}{l}\text { PUENTES } \\
\text { (unidad) } \\
\text { Red } \\
\text { nacional y } \\
\text { red terciaria } \\
\text { a cargo del } \\
\text { INvíAS }\end{array}$} \\
\hline & \multicolumn{2}{|c|}{ A cargo de la Nación } & \multirow{2}{*}{ Subtotal } & & \multirow{2}{*}{$\begin{array}{c}\text { A cargo de la } \\
\text { Nación - INVIAS }\end{array}$} & \multirow{2}{*}{$\begin{array}{l}\text { A cargo de los } \\
\text { departamentos }\end{array}$} & \multirow{2}{*}{$\begin{array}{l}\text { A cargo de los } \\
\text { municipios }\end{array}$} & \multirow{2}{*}{ Subtotal } & & \\
\hline & $\begin{array}{c}\text { Concesionado } \\
\text { - ANI }\end{array}$ & $\begin{array}{c}\text { No } \\
\text { concesionado } \\
\text { - INVAS } \\
\end{array}$ & & & & & & & & \\
\hline 2003 & 0 & 16.528 & 16.528 & 34.918 & 240 & ND & 65.653 & 65.893 & 117.339 & 2.296 \\
\hline 2004 & 0 & 16.677 & 16.677 & 34.918 & 145 & ND & 72.761 & 72.906 & 124.501 & 2.296 \\
\hline 2005 & 0 & 16.750 & 16.750 & 34.918 & 145 & ND & 72.761 & 72.906 & 124.574 & 2.296 \\
\hline 2006 & 2.628 & 14.143 & 16.771 & 34.918 & 145 & ND & 72.761 & 72.906 & 124.595 & 2.296 \\
\hline 2010 & 5.680 & 11.463 & 17.143 & 38.315 & 27.577 & 21.469 & 86.633 & 135.679 & 191.137 & 2.314 \\
\hline 2011 & 5.578 & 11.320 & 16.898 & 42.954 & 27.577 & 13.959 & 100.409 & 141.945 & 201.797 & ND \\
\hline 2012 & 5.262 & 11.856 & 17.118 & 43.327 & 27.577 & 13.959 & 100.409 & 141.945 & 202.390 & ND \\
\hline 2013 & 5.202 & 11.835 & 17.037 & 44.399 & 27.577 & 13.959 & 100.419 & 141.955 & 203.392 & 3.947 \\
\hline
\end{tabular}

Tabla 3 - Información de la red vial de Sucre según el Plan Departamental 
La tabla anterior evidencia la grave situación de la red vial de carreteras en Colombia ya que solo el 8\% (17.037 Km) está categorizada como carreteras primarias las cuales según los criterios están clasificadas como las principales carreteras del país, si analizamos por responsabilidad de los $203.392 \mathrm{Km}$ de carreteras en Colombia a cargo de la Nación, 19,7\% $(39.412 \mathrm{Km})$ son administrados por INVIAS, el 2,5\% (5.202 Km) son administrados por la ANI bajo contratos de concesión y el $78 \%$ restante $(158.645 \mathrm{Km})$ son administrados por los Departamentos y Municipios. En la figura 1 se muestra la distribución de la Red Vial por categorías y por competencias a 2010.

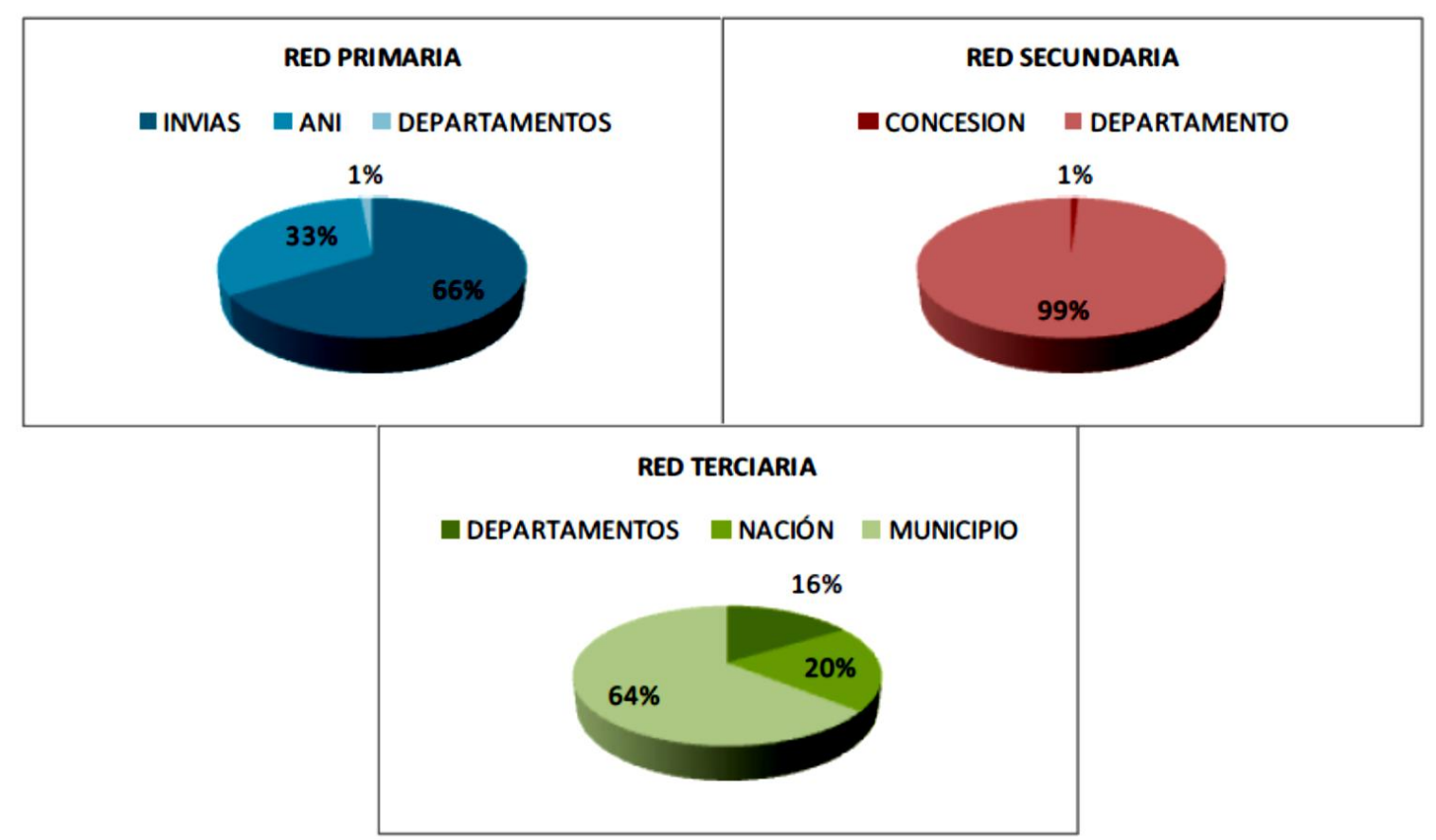

Fig. 1 - Red vial de Colombia por categorías y competencias

Los datos mostrados evidencian que las entidades creadas por el ministerio de transporte de Colombia (INVIAS y ANI) gestionan aproximadamente el $20 \%$ de las carreteras del país encargándose principalmente en la red primaria, esto ha generado problemas de diferentes índoles de la red vial secundaria y terciaria en aspectos como mantenimiento, desarrollo, señalización y modernización, ya que los Municipios y Departamentos tienen menos presupuesto que el gobierno central. Cabe resaltar que las carreteras secundarias y terciarias son las que permiten la comunicación interna entre las regiones.

\subsection{Información técnica disponible para la planificación del transporte en Sucre}

Al igual que en el resto de Departamentos de Colombia, la red vial de Sucre está administrada y gestionada por INVIAS, ANI, Gobernación y Alcaldías Municipales. Esta división de la red vial entre diferentes administraciones estatales en una de las principales razones de la deficiente información técnica del total de las vías del Departamento. Esta afirmación se argumenta en el hecho de que al consultar los informes de las vías elaborados por estas administraciones se encuentran datos contradictorios. En la en la tabla 4 se muestra 
la clasificación de las carretas del Departamento de Sucre según su Plan Vial Departamental 2010-2019 y en la tabla 5 se muestra la red carreteras primarias pavimentadas por departamentos la cual se tomó del anuario estadístico del ministerio de transporte de 2013.

\begin{tabular}{||l|c|c|c|c||}
\hline $\begin{array}{c}\text { JERARQUÍA DE RED } \\
\text { VIAL }\end{array}$ & PAVIMENTADA & AFIRMADO & $\begin{array}{c}\text { EN } \\
\text { TIERRA }\end{array}$ & TOTAL \\
\hline Primaria & 412,4 & 64,8 & & 477,2 \\
Secundaria & 76,8 & 171,1 & 109,8 & 357,7 \\
\hline Terciaria INVIAS & $\mathbf{4 7 , 8}$ & 403,9 & 75,2 & 526,9 \\
\hline Terciaria Departamento & 8,1 & 135,3 & 7,9 & 151,2 \\
\hline Terciaria Municipios & 24,3 & 382,0 & 394,6 & 800,9 \\
\hline \multicolumn{1}{|c|}{ T O T A L } & $\mathbf{5 6 9 , 5}$ & $\mathbf{1 . 1 5 7 , 0}$ & $\mathbf{5 8 7 , 5}$ & $\mathbf{2 . 3 1 4 , 0}$ \\
\hline
\end{tabular}

Tabla 4 -Información de la red vial de Sucre según el Plan Departamental

\begin{tabular}{|l|c|c|c|c|c|c|}
\hline \multirow{2}{*}{$\begin{array}{c}\text { ESTADO DE LA RED } \\
\text { TERRITORIAL }\end{array}$} & \multicolumn{5}{|c|}{ RED PAVIMENTADA $(\mathrm{km})$} \\
\cline { 2 - 7 } & MUY BUENO & BUENO & REGULAR & MALO & MUY MALO & TOTAL \\
\hline Antioquia & 45,28 & 256,99 & 350,32 & 227,49 & 0,00 & 880,09 \\
\hline Atlántico & 43,15 & 28,59 & 13,01 & 9,00 & 0,00 & 93,75 \\
\hline Bolívar & 83,29 & 89,66 & 17,69 & 5,48 & 1,00 & 197,12 \\
\hline Boyacá & 62,73 & 145,10 & 137,80 & 127,20 & 25,21 & 498,04 \\
\hline Caldas & 103,54 & 67,10 & 73,66 & 24,75 & 0,00 & 269,05 \\
\hline Caquetá & 67,19 & 26,82 & 109,86 & 152,34 & 0,95 & 357,17 \\
\hline Casanare & 0,00 & 464,46 & 167,40 & 76,40 & 0,00 & 708,26 \\
\hline Cauca & 44,54 & 224,15 & 159,12 & 79,60 & 5,00 & 512,41 \\
\hline Cesar & 0,00 & 281,21 & 23,62 & 15,00 & 0,00 & 319,83 \\
\hline Córdoba & 89,67 & 152,62 & 61,50 & 27,27 & 4,93 & 335,99 \\
\hline Cundinamarca & 35,74 & 51,60 & 89,26 & 52,09 & 0,08 & 228,77 \\
\hline Chocó & 45,22 & 19,67 & 62,65 & 0,00 & 0,00 & 127,54 \\
\hline Guajira & 0,00 & 66,83 & 65,81 & 1,14 & 0,00 & 133,78 \\
\hline Huila & 110,52 & 102,74 & 177,10 & 137,85 & 1,00 & 529,21 \\
\hline Magdalena & 8,29 & 24,31 & 13,46 & 12,83 & 0,00 & 58,89 \\
\hline Meta & 81,00 & 79,90 & 291,90 & 20,81 & 44,00 & 517,61 \\
\hline Nariño & 186,11 & 224,79 & 73,01 & 121,87 & 0,00 & 605,78 \\
\hline Norte de Santander & 76,14 & 129,78 & 48,08 & 117,63 & 5,96 & 377,60 \\
\hline Ocaña & 0,00 & 41,10 & 26,99 & 78,36 & 1,97 & 148,42 \\
\hline Putumayo & 100,22 & 62,15 & 56,46 & 10,84 & 0,00 & 229,67 \\
\hline Quindio & 3,12 & 33,65 & 41,77 & 10,50 & 0,00 & 89,04 \\
\hline Risaralda & 71,86 & 49,99 & 28,53 & 15,63 & 0,00 & 166,01 \\
\hline S. Andrés y Provid. & 0,00 & 15,50 & 23,80 & 6,00 & 0,00 & 45,30 \\
\hline Santander & 39,04 & 429,48 & 171,87 & 105,08 & 1,38 & 746,85 \\
\hline Sucre & 14,94 & 79,66 & 17,27 & 15,75 & 0,99 & 128,61 \\
\hline Tolima & 48,69 & 171,46 & 67,66 & 14,28 & 1,01 & 303,09 \\
\hline Valle del Cauca & 0,00 & 152,70 & 133,86 & 36,86 & 0,00 & 323,43 \\
\hline \multicolumn{1}{|c|}{ TOTAL } & 1360,28 & 3472,01 & 2503,46 & 1502,06 & 93,49 & 8931,29 \\
\hline
\end{tabular}

Tabla 5 - Red de carreteras primarias por departamento

Realizando una simple comparación entre las dos tablas se puede ver la gravedad del 
problema en cuanto a la información disponible, según la Gobernación del Departamento de Sucre, INIVIAS no tiene vías primarias a su cargo, pero, en la tabla 3 el ministerio muestra que por medio de INVIAS el estado administra $129 \mathrm{Km}$ de vías en el Departamento de Sucre.

Adicionalmente al problema de la información contradictoria entre las administraciones estatales, también se puede ver en la tabla 3 que existen diferentes categorizaciones acerca del estado de las vías terrestres en el País. En todos los informes presentados por INVIAS el criterio técnico empleado para categorizar el estado de la red vial nacional se resume en "Muy Bueno, Bueno, Regular, Malo y Muy Malo", Como se muestra en la figura 2.
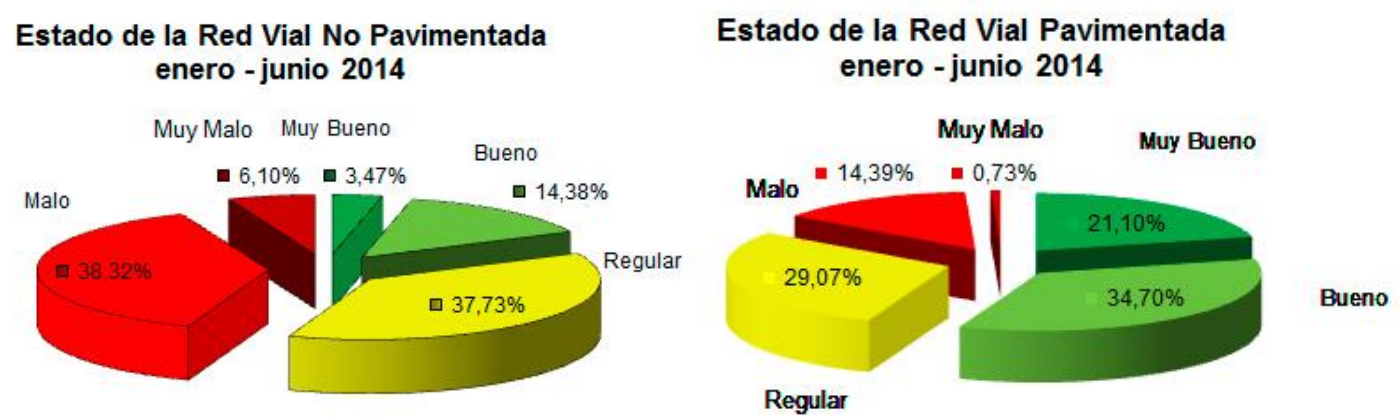

Fig. 2 - Categorización del estado de las vías en Colombia

Este tipo información que sumista el Ministerio de Transporte resulta insuficiente al momento de intentar calcular el tiempo medio de transito de un vehículo entre un origen y un destino determinado, ya que no realiza un mayor aporte a la planeación estratégica del transporte a nivel nacional. Con respecto al departamento de Sucre la situación es aún más compleja ya que más del 50\% de las vías del Departamento están a cargo de Gobernación y Municipios y estas entidades no promocionan ningún tipo de información del estado de las vías, en la figura 3 se muestra el mapa vial de Sucre según las categorías y competencias.

Para complementar la información mostrada en la figura cabe aclarar que la infraestructura vial del Departamento de Sucre tiene una longitud de $2.314 \mathrm{Km}$, de los cuales el $20.6 \%$ $(447.2 \mathrm{Km})$ de la red total corresponden a vías primarias, el $15.5 \%(357.7 \mathrm{Km})$ a vías segundarias y el $63.9 \%(1479 \mathrm{Km})$ a vías terciarias. De los cuales una parte está a cargo de INVIAS, otras se encuentran bajo el formato de concesión vial a cargo de la ANI y el resto son responsabilidad del Departamento y los Municipios. Particularmente INVIAS es responsable de $129 \mathrm{~km}$ que comunican los municipios de Coveñas y San Onofre, teniendo como inicio de esta vía el corregimiento de Sabaneta en el Departamento de Córdoba, recorriendo los municipios de Santiago De Tolu, Tolú Viejo, hasta llegar al municipio de María La Baja en el departamento de Bolívar. La parte de las vías Sucreñas que se encuentra bajo concesión por parte de la ANI son $178 \mathrm{~km}$ comprendidos entre los municipio de Montería en Córdoba y Tolú Viejo en Sucre y un pequeño tramo entre el municipio de San Marcos y Achi según el anuario estadístico del ministerio de transporte del 2013. 


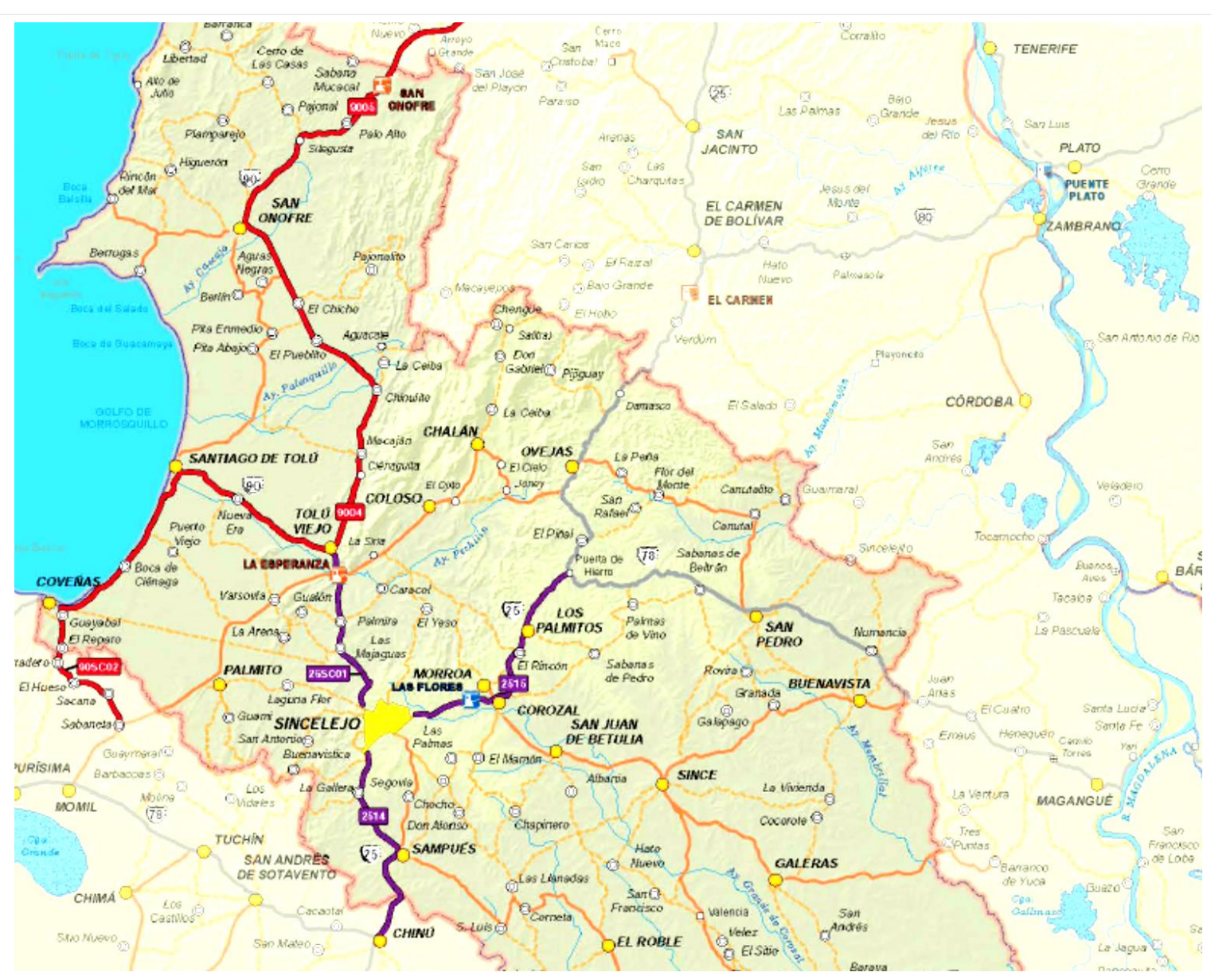

Fig. 3 - Mapa de la red vial de Sucre

La falta de información técnica de la red vial nacional, ha generado un delicado desequilibrio entre generadores y prestadores de servicios de transporte, puesto que el $80 \%$ de los servicios de transporte se realizan por el modo carretero, en donde la desinformación de las condiciones reales, afecta los fletes y costos del transporte por el deterioro de los vehículos y la demora en la entrega de los productos, (Ministerio de transporte, 2010).

A pesar de las ventajas estratégicas que posee el Departamento de Sucre por su ubicación geográfica, que lo sitúa como nodo de paso obligado entre los centros de producción del sur occidente del País y los puertos del Caribe, éstas ventajas no se han potenciado adecuadamente, debido a la ausencia de diagnósticos técnicos reales sobre la infraestructura vial, necesarios para contar con redes intermodales en óptimas condiciones.

Lo anterior también repercute negativamente el crecimiento económico de Sucre basado en su potencial agropecuario, ganadero, cultural y turístico de las diferentes subregiones, ya que por la falta de información no se realizan planes de transporte eficientes que permitan a los empresarios de los diferentes sectores ser competitivos en el mercado nacional e internacional. 


\section{EVALUACIÓN DE RED DE CARRETERAS PRIMARIAS DEL DEPARTAMENTO DE SUCRE}

Para este trabajo se tomó como muestra los tramos de la red de carreteras primarias del departamento que comunican el occidente del país con el puerto de Cartagena, al ser el principal puerto del país, lo anterior comprende tramos de la Ruta 25 que va desde la frontera con el Ecuador (puente de Rumichaca) hasta el departamento del Atlántico (puerto de Barranquilla) y la ruta 90 que va desde la frontera con Panamá (Antioquia) y la frontera con Venezuela (Guajira), Cabe resaltar que la ruta 25 conecta las principales ciudades del occidente Colombiano las cuales representan el $40 \%$ del PIB nacional y que el puerto de Cartagena es el principal puerto del Caribe Colombiano. Para lo anterior se tuvieron en cuenta los datos disponibles por INVIAS, ANI y la gobernación del departamento de Sucre. En la tabla 5 se comparan los datos disponibles con la toma de datos realizadas durante la investigación.

\begin{tabular}{|c|c|c|c|c|c|c|}
\hline \multirow{2}{*}{$\begin{array}{l}\text { Ruta o } \\
\text { código }\end{array}$} & \multirow{2}{*}{ Tramo } & \multicolumn{3}{|c|}{ Longitud en Kilómetros } & \multirow{2}{*}{$\begin{array}{c}\text { Kilometro } \\
\text { Verifi. }\end{array}$} & \multirow{2}{*}{$\begin{array}{c}\text { Diferencia } \\
\text { Máxima }\end{array}$} \\
\hline & & INVIAS & ANI & Adm. Local & & \\
\hline 9004 & $\begin{array}{l}\text { Coveñas } \\
\text { San Onofre }\end{array}$ & 74,57 & N.A. & 74,63 & 75,3 & 0,73 \\
\hline 9005 & $\begin{array}{l}\text { San Onofre - } \\
\text { María la Baja }\end{array}$ & 38,95 & N.A. & 38.9 & 39,4 & 0,5 \\
\hline $90 \mathrm{SC} 02$ & $\begin{array}{l}\text { Coveñas } \\
\text { Sabaneta }\end{array}$ & 15,73 & N.A. & 16 & 16,2 & 0,47 \\
\hline $25 \mathrm{SC} 01$ & $\begin{array}{l}\text { Sincelejo } \\
\text { Toluviejo }\end{array}$ & N.A. & 18 & 18,05 & 22,8 & 4,8 \\
\hline 2514 & $\begin{array}{l}\text { Chinú } \\
\text { Sincelejo }\end{array}$ & N.A. & N.D. & 20,41 & 22,3 & 1,89 \\
\hline
\end{tabular}

Tabla 5 - Longitud de las carreteras primarias de Sucre por entidad

La columna 6 de la anterior tabla muestra los kilometros verificados mediante el uso de un dispositivo GPS utilizando los puntos de inicio y fin por cada tramo según el ministerio de transporte. La tabla evidencia uno de los principales inconvenientes en la gestión de la red de carreteras en el país, ya que los datos suministrados por las entidades estatales no coinciden y en el caso de las carreteras administradas por la ANI la diferencia es aún mayor.

La diferencia más grande se encontró en la carretera 25SC01 administrada por la ANI con 4,8 kilómetros, este tramo se une con la ruta 9004 administrada por INVIAS la cual permite que los vehículos que vienen del occidente del país continúen su viaje hacia la ciudad de Cartagena y por ende la carga al puerto de esta ciudad. En la figura 4 se muestra esta intersección según el mapa del ministerio de transporte a 2013. 


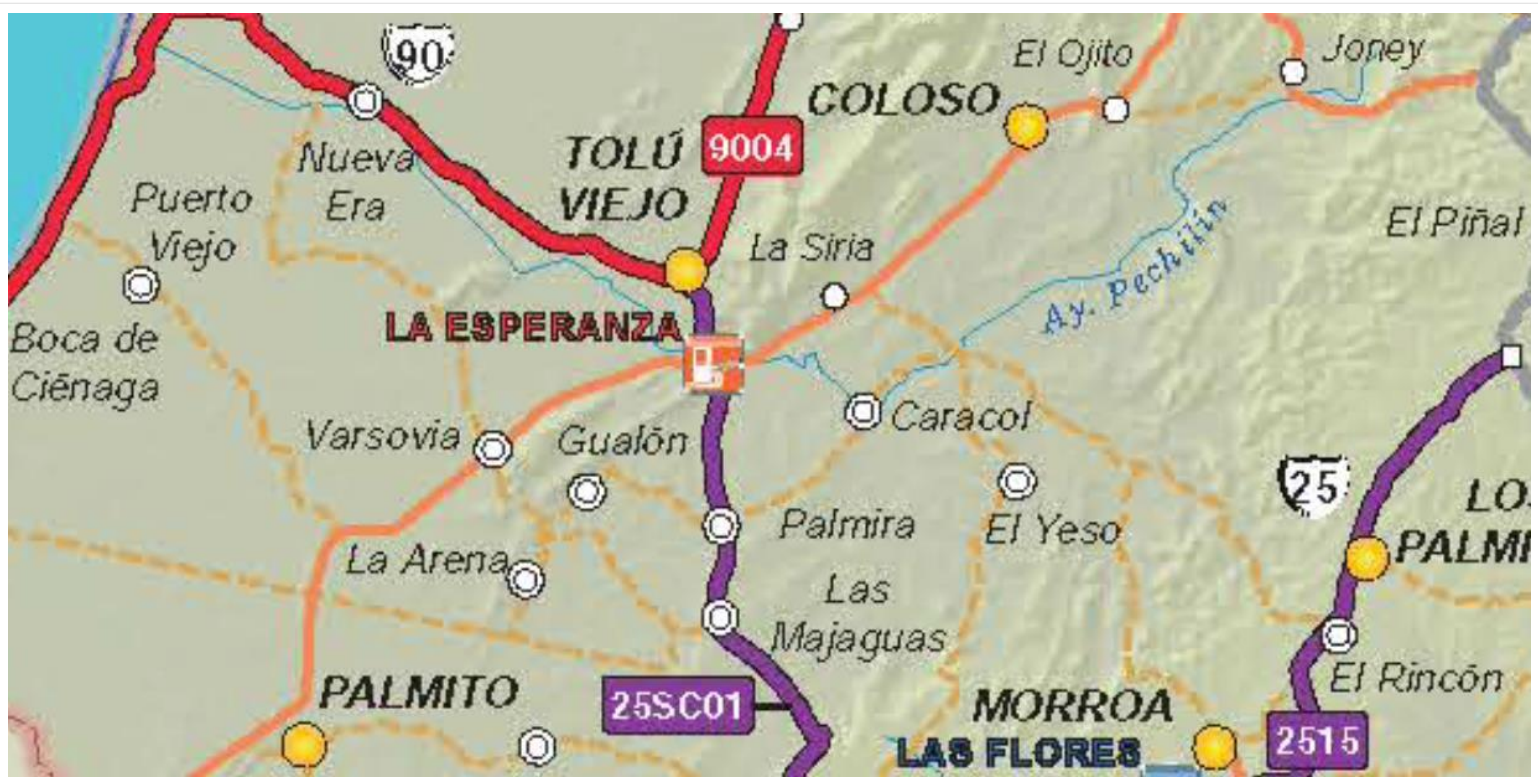

\section{Fig. 4 - Mapa de la red vial de Sucre}

Este tipo de intersecciones en las que se unen carreteras administradas por diferentes entidades generan inconsistencias en la cantidad de kilómetros que debe administrar cada entidad lo cual genera vacíos jurídicos que se derivan en descuido en la gestión de ciertos tramos de la red vial y por ende se ocasionan situaciones tales como:

- Deficiente señalización horizontal (aquella que se pintan sobre el pavimento)

- Inexistente señalización vertical

- Viviendas a menos de un metro de la carretera

- Irregularidades en el asfalto

- No existen barandas de protección

En la figura 5 se muestra la imagen de la actual intersección de Toluviejo.

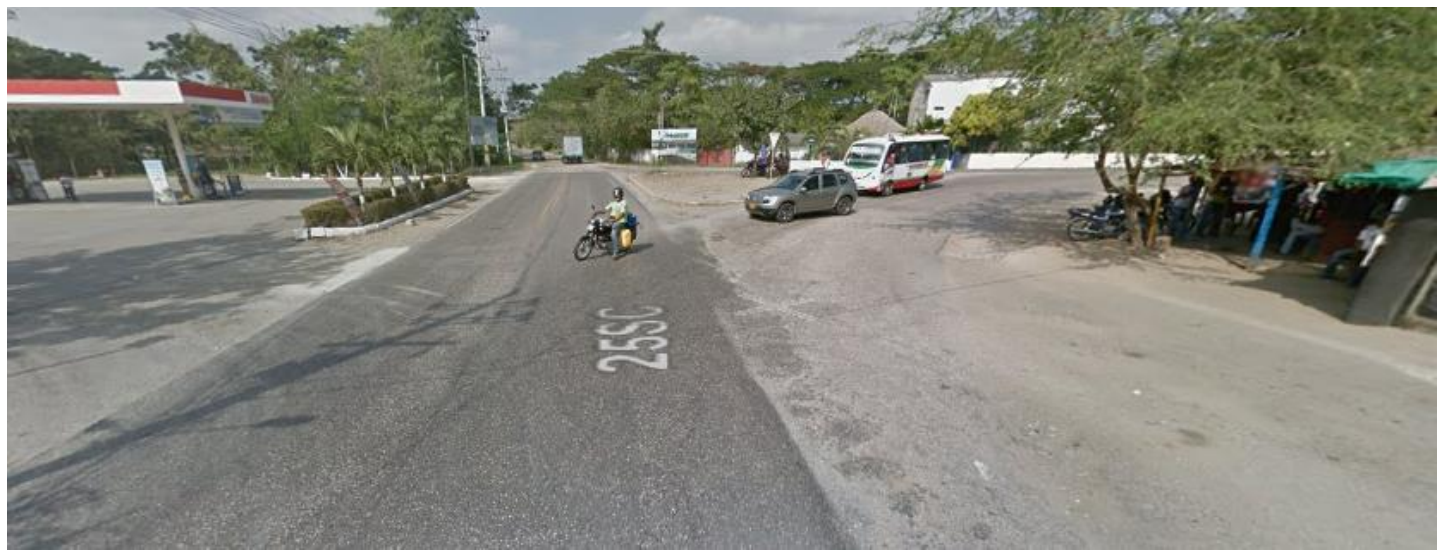

Fig. 5 - Intersección Toluviejo 
Situaciones como las que se presentan en la intersección de Toluviejo, en las cuales es evidente que la red de carreteras del País presentan graves problemas, generó que el ministerio de transporte realizará un diagnóstico de las carreteras pavimentadas, según el documento CONPES 3085 del año 2000, este diagnóstico se realiza por observación visual y dio como resultado tres calificaciones las cuales son:

- Vías en buen estado: aquellas que únicamente requieran un mantenimiento rutinario.

- Vías en regular estado: aquellas que requieran renovación o refuerzo de su superficie

- Vías en mal estado: aquellas que requieran rehabilitación o reconstrucción inmediata.

Como se puede ver en la figura 2 y en la tabla 4 el ministerio muestra la crítica situación de la red de carreteras del país al tener cerca del 50\% con calificación de regular y malo, para analizar el impacto que tiene esto en la duración de los tiempos de viaje, se midieron estas variables en los tramos estudiados, los resultados obtenidos se muestran en la tabla 6 .

\begin{tabular}{|c|l|c|c|c|c|c|}
\hline $\begin{array}{c}\text { Ruta o } \\
\text { código }\end{array}$ & \multicolumn{1}{|c|}{ Tramo } & $\begin{array}{c}\text { Longitud } \\
\mathrm{km} .\end{array}$ & $\begin{array}{c}\text { Velocidad } \\
\text { máxima }\end{array}$ & $\begin{array}{c}\text { Velocidad } \\
\text { forzada }\end{array}$ & $\begin{array}{c}\text { Velocidad } \\
\text { promedio }\end{array}$ & $\begin{array}{c}\text { Tiempo } \\
\text { de viaje }\end{array}$ \\
\hline 9004 & $\begin{array}{l}\text { Coveña }- \\
\text { San Onofre }\end{array}$ & 75,3 & $70 \mathrm{Km} / \mathrm{h}$ & $40 \mathrm{Km} / \mathrm{h}$ & $50 \mathrm{Km} / \mathrm{h}$ & $90 \mathrm{~min}$ \\
\hline 9005 & $\begin{array}{l}\text { San Onofre - } \\
\text { María la Baja }\end{array}$ & 39,4 & $55 \mathrm{Km} / \mathrm{h}$ & $40 \mathrm{Km} / \mathrm{h}$ & $50 \mathrm{Km} / \mathrm{h}$ & $51 \mathrm{~min}$ \\
\hline $90 \mathrm{SC02}$ & $\begin{array}{l}\text { Coveñas - } \\
\text { Sabaneta }\end{array}$ & 16,2 & $55 \mathrm{Km} / \mathrm{h}$ & $40 \mathrm{Km} / \mathrm{h}$ & $48 \mathrm{Km} / \mathrm{h}$ & $26 \mathrm{~min}$ \\
\hline $25 \mathrm{SC01}$ & $\begin{array}{l}\text { Sincelejo - } \\
\text { Toluviejo }\end{array}$ & 22,8 & $80 \mathrm{Km} / \mathrm{h}$ & $50 \mathrm{Km} / \mathrm{h}$ & $55 \mathrm{Km} / \mathrm{h}$ & $32 \mathrm{~min}$ \\
\hline 2514 & $\begin{array}{l}\text { Chinu } \\
\text { Sincelejo }\end{array}$ & 22,3 & $80 \mathrm{Km} / \mathrm{h}$ & $50 \mathrm{Km} / \mathrm{h}$ & $55 \mathrm{Km} / \mathrm{h}$ & $30 \mathrm{~min}$ \\
\hline
\end{tabular}

Tabla 6 - Tiempos de viaje y velocidad según tramo

Cuando se realizaron los diferentes recorridos fue necesario registrar dos tipos de velocidades, la cuales fueron la velocidad máxima (velocidad máxima que se alcanzó en algunos tramos de la carretera) y velocidad forzada (velocidad en la que se debido transitar en algunos tramos) lo anterior debido a diversas situaciones tales como:

- Mal estado de las carreteras: el mal estado obligaba a disminuir la velocidad para evitar accidentes

- Un solo carril para transitar: al solo existir un carril tanto lo vehículos de carga como los particulares se generan congestiones en la vía

- Poblaciones a menos de un metro de la carretera: existen viviendas, colegios, y otro tipo de construcciones a menos de un metro de la carretera sin barreras de protección lo cual incrementa el riesgo de accidentes y la dificultad en el tránsito. 
Todo lo anterior ocasiona que los tiempos de viaje sean más largos ya que la velocidad de transito promedio esta en los $55 \mathrm{~km} / \mathrm{hora}$, lo cual repercute directamente en los costos de viaje, en la tabla 7 se comparan rutas de viaje en España con rutas de viaje en Colombia

\begin{tabular}{|l|c|c|c|c|c|}
\hline \multicolumn{1}{|c|}{ Tramo } & $\begin{array}{c}\text { Longitud } \\
\mathrm{km} .\end{array}$ & $\begin{array}{c}\text { Número } \\
\text { de carriles }\end{array}$ & $\begin{array}{c}\text { Velocidad } \\
\text { Max }\end{array}$ & $\begin{array}{c}\text { Velocidad } \\
\text { promedio }\end{array}$ & $\begin{array}{c}\text { Tiempo } \\
\text { de viaje }\end{array}$ \\
\hline Madrid - Sevilla & 534 & $2 \times 2$ & $130 \mathrm{Km} / \mathrm{h}$ & $110 \mathrm{Km} / \mathrm{h}$ & $300 \mathrm{~min}$ \\
\hline Medellín - Sincelejo & 468 & $1 \times 1$ & $80 \mathrm{Km} / \mathrm{h}$ & $55 \mathrm{Km} / \mathrm{h}$ & $560 \mathrm{~min}$ \\
\hline Madrid - Barcelona & 621 & $2 \times 2$ & $130 \mathrm{Km} / \mathrm{h}$ & $110 \mathrm{Km} / \mathrm{h}$ & $360 \mathrm{~min}$ \\
\hline Medellín - Cartagena & 638 & $1 \times 1$ & $80 \mathrm{Km} / \mathrm{h}$ & $50 \mathrm{Km} / \mathrm{h}$ & $780 \mathrm{~min}$ \\
\hline Sevilla - Algeciras & 183 & $2 \times 2$ & $130 \mathrm{Km} / \mathrm{h}$ & $110 \mathrm{~km} / \mathrm{h}$ & $110 \mathrm{~min}$ \\
\hline Sincelejo - Cartagena & 170 & $1 \times 1$ & $70 \mathrm{Km} / \mathrm{h}$ & $50 \mathrm{Km} / \mathrm{h}$ & $200 \mathrm{~min}$ \\
\hline
\end{tabular}

Tabla 7 - Comparación tiempos de viaje entre Colombia y España

La tabla anterior demuestra que los tiempos de viaje en Colombia son el doble que los tiempos de viaje en España para distancias iguales, lo que repercute directamente en los costos de transporte en el país. Para calcular los tiempos de viajes de la tabla anterior fue utilizada la herramienta maps de Google ya que este es el único GPS que comparten ambos países a diferencia de Europa donde herramientas como TomTom sirven para todo el continente.

Según el Doing Bussiness, del Banco mundial, el costo de importar o exportar un contenedor de 40 pies en Colombia es de 2.355 USD y 2.470 USD respectivamente, de los cuales en promedio 1.800 USD corresponden al costo del transporte interior. Si analizamos este mismo indicador para el caso de América Latina el costo total de importar o exportar el mismo contendor es de 1.299 USD y 1.691 USD respectivamente. Por ende solo el coste de transporte en Colombia supera el costo total de una operación de comercio exterior en América Latina.

\section{CONCLUSIONES}

Colombia es un país que mueve más del $80 \%$ de su carga por carreteras, según cifras del Departamento Nacional de Estadística DANE, y cuenta con una red de carreteras que suma 203.392 Km según datos del ministerio de transporte, pero solo $17.037 \mathrm{Km}$ que equivale al $8 \%$ está categorizado como red vial de primer orden, por ser esta red de carreteras las que comunican los principales centros de consumo o son paso de frontera, ambos criterios cualitativos más que cuantitativos, porque si analizamos los otros criterios que contempla el ministerio encontramos grandes inconsistencias.

Según la empresa autopista de la Sabana que tiene en concesión un tramo de la ruta 25 que está en el Departamento de Sucre por la vía están transitando más de 20.000 vehículos al día muy por encima de los 700 que pide el ministerio en el criterio técnico que denomina "Transito promedio diario" para considerar una vía como de primer orden, lo anterior 
evidencia la descontextualización de los criterios técnicos del Ministerio con la realidad del transporte por carreta del país.

Es por esto que indicadores de infraestructura como el Foro Económico Mundial sitúan a Colombia en los últimos puestos al igual que el Doing Bussines, pero en este caso analizando costos y tiempos de las operaciones logísticas y comerciales que soportan el comercio exterior del país. Algo consecuente al comprobar que menos del $20 \%$ de la red vial $(39.412 \mathrm{Km})$ está a cargo de la Nación y que de este porcentaje cerca del $40 \%$ se encuentra en mal estado.

Este trabajo como primer acercamiento a la evaluación de la red de carreteras de una de las regiones de Colombia permite concluir que es necesario revisar y actualizar los criterios técnicos con los que se están categorizando las carretas de Colombia, ya que estos no están correspondiendo a el nuevo panorama Mundial impulsado por la globalización y los estándares logísticos internacionales. Como futuras líneas de investigación se plantea analizar la red secundaria y terciaria de esta región con el fin de comprobar si se presentan situaciones similares a las de la red primaria.

\section{AGRADECIMIENTOS}

Este proyecto se realizó con el apoyo de la Corporación Universitaria del Caribe CECAR mediante la convocatoria de proyectos Internos del 2015

\section{REFERENCIAS}

ARDILA, A. (1999) La Planeación Del Transporte: Una Nueva Propuesta Con Énfasis En La Operación Y El Mantenimiento, Revista de Ingeniería, vol 9, 28 - 37.

BALLOU, RONALD (2004) Logística, Administración de la cadena de suministro. Pearson - Prentice Hall.

BARBERO, J. (2013) La infraestructura en el desarrollo integral de América latina. Banco de Desarrollo de América latina, Corporación andina de Fomento CAF.

CALKINS, H. (1990) GIS and public policy. Geographical Information Systems Vol 2.

CHOPRA, SUNIL (2008) Administración de la cadena de suministros-Estrategia, planeación y operación. Bogotá Pearson Educación: Prentice Hall.

Diario oficial de la Republica No. 48.773, viernes 26 de 2013, Imprenta nacional, Bogotá, D.C.

DICKEY, J. (1983) Metropolitan transportation planning. Hemisphere Pub. Co-McGrawHill, New York, Second edition.

Gaceta Departamental de Sucre (2010). Plan Vial departamental de Sucre 2010 - 2019. Sincelejo: Gobernación de Sucre.

GAKENHEIMER, R. (1995) Shaping the future: the role of urban transportation planning. 
China's Urban Transport Development Strategys. The World Bank.

Ley de categorización de la red vial nacional, Articulo 1 de la ley 1228

MCGINNIS, M. (1990) The Relative Importance of Cost and Service in Freight Transportation Choice: Before and After Deregulation, Transportation Journal, Vol. 30, Núm. 1. 12-19.

MILLER, J. Y MEYER, J. (1984) Urban transportation planning: a decisions oriented approach.

Ministerio de transporte. (2010). Plan nacional de desarrollo 2010 - 2014. Sector transporte. Bogotá: ministerio de transporte.

MORENO, G. (2007). Análisis con SIG de la red de transporte intermodal entre Marruecos y la Unión Europea. Evaluación de rutas y enclaves estratégicos.

Plan estratégico intermodal de infraestructura de transporte 2013, Ministerio de Transporte REIS, V., MEIER, J., PACE, G., \& PALACIN, R. (2012). Rail and multi-modal transport. Research in Transportation Economics, 17 - 30.

Transporte en cifras, Anuario estadístico 2013, Ministerio de Transporte

http://ani.gov.co/

http://espanol.doingbusiness.org/

https://www.mintransporte.gov.co/

http://www.dane.gov.co/ 